\title{
XVII.
}

\section{Beschreibung zweier Microcephalen-Gehirne mit einigen Bemerkungen}

von

\author{
Dr. Julius Sander, \\ Assistenzarzt der Nervenklinik des Koniglichen Charite-Krankenhauses \\ zu Berlin.
}

Hierzu Tafel V. und VI.

$\mathrm{I}_{\mathrm{n}}$

die von Herrn Geheimerath Griesinger dirigirte Poliklinik wurde im Beginn der Sommerferien 1867 ein junges Kind gebracht, dessen eigenthumliche Schadelbildung Herrn Dr. Vaneschi sofort auffiel. Derselbe hatte die Freundlichkeit, mich davon zn benachrichtigen, so dass wir gemeinschaftlich die Obduction des Kindes ausfuhren konnten; ich bin ihm auch fur die Notizen über das kurze Leben des Individuoms verpflichtet.

Adolph Pfofteıle wurde am 10. April 1867 geboren. Die Mutter des Kindes, Ottilie Pfefferle, ist an 23. December 1851 geboren. Mit 14 Jahren wurle sie znerst menstruirt; im Juli 1866, also 141/2 Jahre alt, hatte sie mit einem Mann von mittlerer Statur, der zwanzig und einige Jabre alt sein soll, viermal geschlechtlichen Verkehr. Im Januar 1867 fuhlte sie zuerst Kindesbewegungen und am 10. April wurde sie sehr leicht (in 2 Stunden) von einem lebenden Knaben entbunden, der zwar sofort schrie, aber sehr elend aussah. Die Hebeamme ausserte, sie habe noch nie ein Kind mit einem so kleinen Kopf gesehen. Die Mutter stillte das Kind selbst bis 6 Wochen vor seinem Tode; es hatte zwar nach der Geburt geschrieen, war aber spater immer sehr ruhig und nahm gierig Nahrung. Es magerte allmalig ab und starb ohne Symptome einer bestimmten Krankheit am 16. September.

Obduction etwa 30 Stunden p. m, nur die Schidelhöhle konnte geöfnet werden. - Das Kind bot in seinem Aeussern ganz das bekannte Bild des sog. Aztekentypus; es war 62 Centim. Jang; das Gewicht konnte nicht bestimmt werden. Die Muskulatur ist atrophisch, die Knochen sehr dínn, ein Panniculus so gut wie gar nicht vorhanden; die Haut dünn und welk, der Kopf. mit spärlıchen Haaren bedeckt. - Der Schädel ist im Verhaltniss zur Länge des Kindes auffallend klein, langlich, von den Seiten her zusammengeduückt. 
Seine Dimensionen sind folgende: $\left.{ }^{*}\right)$

1) Vom Scheitel bis zur Kinnspitze (über das Gesicht periphernsch). . 151/2 C.

2) Der grosse diagonale Durchmesser von der Kinnspitze bis zur Lambdanaht, der Stelle der kleinen Fontanelle......... 11/2 .

3) Der gerade Lurchmesser von der Glabella bis zum hervorragendsten

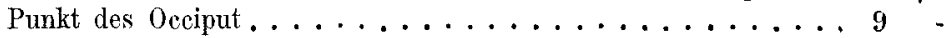

4) Der biparietale ...................... 81/2 -

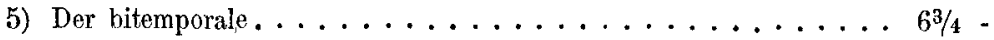

6) Von einem Porus acust. ext. zum andern. ........... 71/2 .

Die Peripherie des Schadels beträgt von der Glabella bis zum hinteren Rande des Foramen magnum $171 / 2 \mathrm{C}$, von einem Ohr quer heruber bis zum andern $16 \mathrm{C}$., die grosste Circumferenz des Kopfes $28 \mathrm{C}$.

Der Schadel ist vollkommen verknochert: die Nähte lassen sich deutich durchfühlen, doch sind die Fontanellen völlig geschlossen. Die Stirn ist niedrig, tritt etwas zurück, dagegen springt die Nase hervor; die Nasenwurzel liegt sehr hoch. Die Linie der Stirn geht gerade in die der Nase uber, so dass das Gesicht den bekannten Ausdruck des Vogelgesichts bekommt. Das Kinn springt wieder etwas hervor; der Mund ist gross; die Ohrmuscheln sind gross mit deutlichen Lappchen, schief inserirt, weit vom Kopfe abstehend, auf beiden Seiten ungleich und unsymmetrisch, das rechte etwas über 4 , das linke $3 \frac{1}{2} \mathrm{C}$. von oben nach unten lang. Die Genitalien sind gut entwickelt, der rechte Hode liegt im Scrotum, der linke befindet sich noch im Leistenkanal. - Die Peripherie des Stammes betragt über den Brustwarzen 30 C.; die -über dem Nabel konnte nicht bestimmt werden, da der Bauch bereits durch Fäulmssgase stark aufgetrieben war. Die Masse der Extremitäten betragen beiderseits ungefihr gleich:

Trochanter major bis Calcaneus ...... $23 \mathrm{C}$.

Trochanter major bis Mitte der Patella .... $11 \mathrm{C}$.

Mitte der Patella bis zum Sprunggelenk ... 101/2 C.

Ober- und Unterarm nahezu gleich lang ... $71 / 2 \mathrm{C}$.

Die Hand ist $5 \mathrm{C}$, der Mittelfinger $23 / 4 \mathrm{C}$. lang. -

Bei der Eröffnung des Schädels, die mit der Säge vorgenommen werden musste, finden sich die Schädelknochen stark mit der Dura mater verwachsen; die Pia mater zeigt viele stark gefïllte Gefässe; die Hurnsubstanz ist sehr weich. Die grösste Breite der hinteren Schddelgrube betragt 51/2 C., der mittleren $7 \mathrm{C}$, , der vorderen $4 \frac{1}{2}$ C.; die Lange der ganzen Schadelbasis betragt $8 \mathrm{C}$. Die Verkúmmerung ist am stärksten in den vorderen Schadelgruben ausgesprochen. Die Nähte an der Basis sind gut erhalten, der Turkensattel ist gut gebildet. Die Vertiefungen zu beiden Seiten der Crista galli dringen sehr tief ein. Die Schádelknochen erscheinen mássig blutreich.

Das Gehim, das leider nur mit schlimmen Verletzungen aus dem Schadel hatte herausgenommen werden konnen, wog frisch nicht ganz $170 \mathrm{Grm}$. Messungen waren an dem frischen Gehirn nicht ausfuhrbar; jetzt, nach vorsichtiger Erhärtung, beträgt**) die Lánge von der Spitze des Stirnlappens his zur Spitze des

*) Die Messungen mussten in Eile ausgefuhrt werden und ohme dass die Weichtheile ganz entfernt werden konnten, da es nicht moglich war, den Kopf zu erwerben.

${ }^{* *}$ ) Die Messungen sind an der besser erhaltenen linken Hemisphare ausgeführt, die auch allein abgebildeł ist. Die rechte zeigte jedoch kaum Unterschiede. 
Hinterlappens, quer uber die laterale Flache der Hemisphäre gemessen, $9^{3 / 4} \mathrm{C}$. Die Distanz von der Spitze des Stirnlappens bis zur Rolando'schen Spalte (Fig. I., I.) làns des oberen Randes $63 / 1$ C., bis zur vorderen Centralwindung (a) 5 C. Die Hòhe des Stirnlappens vor der vorderen Centralwindung beträgt $3^{1 / 2}$ C. Eine von dem oberen Ende der Centralfurche bis zur Spitze des Klappdeckels $(\alpha)$ gezogene Linie misst 31/4 C. Von der Spitze des Stirnlappens bis zum Hinterhauptspalt (II.) 10 C. lángs des oberen Randes an der lateralen Mantelfläche. Die Lánge des Hunterlappens selbst beträgt noch nicht $1 \mathrm{C}$, seine Höhe $3 / 4 \mathrm{C}$. Von der Spitze des Schlafenlappens bis zur Scissura pallii misst man parallel der Fissura parallela (III.) $71 / 2$ C. Die grösste Lảnge der Hemisphare beträgt an der medialen Wand $8 \mathrm{C}$., ihre grósste Höhe daselbst $31 / 2 \mathrm{O}$.

Die Windungen sind durchweg verhältnissmässig breit und plump; dı Sulci dringen wemg in die Tiefe, Nebenwindungen sind kaum vorhanden. Die vordere Centralwindung (a) geht als grader Windungszug herunter, direct in die hintere (b) umbiegend, die ihrerseits aus 2 Theilen besteht, von denen der obere (c) in den 1., der untere (b) in den 2. Parletalwindungszug (ph courbe 7) sich fortsetzt und in die Wurzel des 1. gyrus sphenoidalis (4). Der Klappdeckel ist demgemäss klein und reicht kaum so weit herunter, wie beim normalen Menschengehirn. Vor der Spitze 'des Schlafenlappens geht eme Windung (3) die bei $\boldsymbol{a}$ durch eine schmale, von einer seichten Furche durchbrochene Brücke mit der vorderen Centralwindung zusammenhängt, senkrecht von oben nach unten; s1e schlägt sich vor der Gegend der Substantia perforata antica um in Aie 3. Stirnwindung. Die vordere Begranzung der Fossa Sylvu wird demnach hier von der 3. Stirnwindung gebildet im Gegensatz zu den Angaben C. Vogt's, auf die ich später ausfuhrlich zuruckkomme. Ganz oben an der Scissura palli schlagt sich de vordere Centralwindung herum in die 1. Strrnwindung (1.), die aus 2 parallelen Windungszugen besteht. Auch die 2. Stirnwindung (2.) wird von 2 parallelen Windungen gebildet, die nur in ihren vordersten Theilen in Verbindung treten mit der 1. und 3. Am Lobulus orbitalis erschemen die Wondungen nur wenig ausgeprägt; ein Siebbemschnabel ${ }^{*}$ ) war sicher stark ausgebld st, ist aber am gehärteten Gehirn nicht mehr zu sehen. Die Gyri breves der Insel sind kaum angedeutet. Dic hintere Centralwindung geht, wie erwihnt, direct liber in de 2 Parietalwindung (7) und in die Wurzel des oberen Gyrus sphenoidalis, oben in den Vorzwickel (e). Ebenso gehen auch die Wurzeln des 2. und 3. Gyrus sphenoidalis (5 und 6) direct uber in verhältnissmässig reich gefaltete Windungen des Scheitellappens (e und f). Die gyrı sphenoidalis selbst sind sehr einfach; der ganze Schläfenlappen ist conform den Angaben Vogt's (1 c. p. 225) im Verhältniss zum Grosshirn gross. Der Vorzwickel (e, f) ist gross; der Zwickel (g) klein. An der lateralen Flàche der Hemisphare sind andere Windungen des lobus occipitalis eigentlich gar nicht vorhanden; nur an der medialen Seite sind einige von dem gut entwickelten gyrus fornicatus (Fig. II., $g$ forn.) in seinen hintersten Theilen nach oben ziehende Windungen $(h, 1)$ noch zum Hinterlappen zu rechnen.

Das Cerebellum ist fast bedeckt rom Grosshirn. Die linke Hemisphäre (die

*) cf. C. Vogt: Ueber die Microcephalen etc. Archiv für Anthropologie II., 2. p. $228 \mathrm{ff}$. Ich habe übrigens in letzter Zeit seitdem ich auf diese Verhaltnisse achte, gar nicht selten sehr stark ausgebildete Siebbeinschnabel bei Sectionen von Europäern gesehen, deren Gehirn sonst in jeder Beziehung normal entwickelt war. 
rechte war so stark beschädigt, dass sie fur die Untersuchung unbrauchbar geworden) ist $3 \frac{1 / 2}{} \mathrm{C}$. lang in maximo $1^{3 / 4} \mathrm{C}$. breit und $1 \frac{1 / 4}{\mathrm{O}}$. hoch. Der obere Wurm ist $21 / 2$ C. lang. - Die Theile der Basis sind gut entwickelt, nur das Corpus candicans ist kaum angedeutet. Die Brücke hat eine Lange von $7 / 8 \mathrm{C}$. Die Vierhuigel verhalten sich normal. Der Thalamus opticus (th. 0.) ist $11 / 2 \mathrm{C}$. hoch, die stria semicircularis und die Decke des 3. Ventrikels, die tela choreoidea superior sind gut ausgebildet, ebenso der Trichter. Der Seitenventrikel (rechts) ist durchaus nicht erweitert, seine Decke wird in normaler Weise vom Balken (c. call.) gebildet, der $3^{1} / 2$ C. lang ist; während das Knie (gen.) eine Höhe von $1 \frac{1}{2}$ C. zeigt, ist das Splenium (spl.) auf eine dünne Lamelle reducirt, von der man als ganz dinne Platten die Balkenstrahlung (Zange und Tapete) in den Hinter- und Schlafelappen einstreichen sieht. Das Vorderhom reicht vom vorderen Rande des Thalamus $3 / 4 \mathrm{C}$. nach vorn; das absteigende Horn dringt fast $2 \mathrm{C}$. weit nach unten vor; das Hinterhorn ist nur angedeutet. Der Boden des Seitenventrikels wird in normaler Weise vom Corpus striatum und dem Fornix (forn.) gebildet; letzterer ist aber sehr schwach, ebenso wie das Cornu Ammonis, das sich als eine dünne Lamelle darstellt, aber deutliche Klauen zeigt Das Septum pellucidum (s. l.) ist in gewòhnlicher Weise vorhanden. Das Quercommissurensystem des Grosshirns beginnt in normaler Weise an der lamina terminalis (l. t.) und schreitet auch bis zum Splenium vor, nur ist letzteres zu schwach ausgebildet. Die Commissura anterior (C. a.) ist vorhanden, die C. mollis fehlt. Die Fasern der Zwinge sind gut entwickelt, nur der Iaken erscheint schwach. Ueber das Verhalten der einzelnen Kerne des Stammlappens lasst sich nichts Bestimmtes ermitteln; wahrschemlich verhalten sie sich normal.

Der Liberalitat meines verehrten Lehrers, des Herrn Geheimerath Reichert, verdanke ich es, dass ich hier noch die Beschreibung und Abbildung des auf dem hiesigen anatomischen Museum unter No. 12590 autbewahrten Gehirns des bekannten Friedrich Sohn hinzufügen kann. Die Geschichte dieses Microcephalen ist hinreichend ausfuhrlich oftmals mitgetheilt worden, zuerst von Johannes Müller selbst (Medicinische Zeitung des Vereins fur Heilkunde in Preussen, 1836, No. 2 und 3); ich kann sie daher übergehen; Abbildungen des Schädelausgusses befinden sich bei $\mathrm{C}$. Vogt, l. c. tab. X.

Das Gehirn wiegt 29 Loth. Die wichtigsten Masse sind folgende:

Länge der linken Hemisphäre....... 141/4 C. rechten $" \quad \ldots \ldots \ldots . . .141 / 2$ C.

Grosste Breite des linken Vorderlappens ... 2 $2^{3 / 4}$ C.

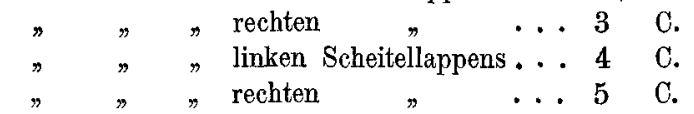

Die Rolando'sche Centralspalte ist rechts 9 C. von der Spitze des Stirnlappens entfernt, links $8 \mathrm{C}$.

Eine Hinterhauptsspalte ist an der lateralen Mantelfläche mit Sicherheit gar nicht zu bestimmen; man würde ganz willkürlich irgend einen der hinteren Sulci als solche bezeichnen müssen. (Auf Vogt's Tafel figurirt als Hinterlappen [D.] 
ein Theil, der zum Cerebellum gehort). Die rechte Hemisphare ist grösser; an der rechten liegt auch die Centralfurche (I.) weiter zurück, als an der linken; die Anordnung der Windungen ist gleichwohl ziemlich symmetrisch. Die vordere Centralwindung (a) steigt ziemlich gerade und glatt herunter, hängt nach vorn mit der 3. Stirnwindung (3), nach hinten mit der hinteren Centralwindung (6) zusammen. Der Klappdeckel reicht auch hier keineswegs so weit herunter, dass er die vordere Begränzung der Fossa Sylvii ausmachte; mag man nun die Gegend bei $\boldsymbol{\alpha}$ (Fig. IV.) oder bei $\beta$ als seine Spitze annehmen, jedenfalls betheiligt sich noch die 3. Stirnwindung, die ubrigens durch eine Brucke mit der vorderen Centralwindung zusammenhängt, ziemlich bedeutend. Die Stirnwindungen sind sehr einfach und plump; die 1. Stirnwindung (1.) entspringt direet aus der vorderen Centralwindung, alle drei hängen unter einander zusammen. Auch hier ist der Schläfenlappen verhältnissmȧssig gross. Die Fissura parallela (11) ist sehr deutlich; dagegen ist der 3. Gyrus sphenoidalis (6) nur sehr wenig vom 2. (5.) geschieden; die hintere Centralwindung (b) ist mehrfach gekerbt und geht oben direct in den Vorzwickel (e), unten in die Wurzel des 1. Gyrus sphenoidalis (4) und in die Gegend des 2. Parietalwindungszuges (pli courbe, $f$ ) über. Auch die beiden andern gyri sphenoidales entspringen vom Scheitellappen. Ein kleiner Zwickel (g) ist zwar allenfalls vorhanden, sonst vermag ich an der peripheren Mantelfache keine gyri occipitales aufzufinden. Die Srebbenschnábel sind hier nur mássig ausgebildet; die gyru des lobulus orbitalıs und die gyn breves sind kummerlich entwickelt. Die mediale Mantelfliche konnte nicht untersucht werden. -

Das Cerebellum (E) 1st jederseits $6^{\frac{1}{2}} \mathrm{C}$. lang und $3^{1 / 2} \mathrm{C}$. breit. Der Balken 1st etwas kurzer, als er bel normalen Gehirnen zu sein pflegt, so dass die hinteren Vierhigel von shm unbedeckt bleiben. Auch hier kann man sich uberzeugen, dass das Splenium des Balkens unverháltnssmassig diunn ist. (Da kein Schnitt durch das Gehirn gelegt werden durfte, so kann von diesem Verhalten keine Abbildung gegeben werden). Die Riechkolben sind sehr schmal und lang, die übrigen Nerven der Basis sonst sammtlich ziemlıch stark. Die Hypophysis jst gross. Die Brucke ist $1 \frac{1}{2} \mathrm{C}$. lang, $21 / 2 \mathrm{C}$. breit; die Medulla oblongata ist $2 \mathrm{C}$. breit.

Ueber Microcephalen ist schon so viel gesehrieben worden und die Litteratur findet sich in der citirten Schrift von C. Vogt so vollstandig zusammengestellt, dass ich mich hier darauf beschranken kann, nur einzelne Punkte, in denen meine Untersuchungen mich zu anderen Resultaten geführt haben als denen des eben genannten berühmten Forschers, naher zu betrachten. Die Affenähnlichkeit dieser Individuen ist eine so frappante, dass sie natürlich jedem, der in die Lage kam, genauere Untersuchungen über sie anzustellen, sich aufdrängen musşte. Dieser Umstand wurde von den Anhängern der Descendenztheorie Darwin's mit Freuden begrusst, während die Gegner der Theorie sich alle erdenkliche Muhe gaben, nachzuweisen, dass diese Aehnlichkeit nur eine äusserliche sei. Prüfen wir die Sachlage für das Gehirn, so stehen sich hier schroff zwei Ansichten gegenüber. Rudolph Wagner (Vorstudien etc., zweite Abhandlung, Göttingen 1862 p. 83) sagt: „Die Microcephalen-Gehirne stellen keinen Rückfall in den Affentypus, keine 
Verahnlichung mit demselben dar. Wahrend sie im vorderen Theile der Hemisphären, der grösseren Einfachheit wegen, sich den Gehirnen der hoheren Affen allerdings nähern, entfernen sie sich im hinteren Theile davon um so mehr." Er stutzt sich fur diesen Schluss wesentlich auf die hochgradige Verkúmmerung der lobi occipitales, wahrend bei den Affen grade diese Lappen besonders gut entwickelt und durch einen tiefen Sulcus occipitalis anch an der lateralen Mantelflache von dem Scheitellappen abgesetzt sind. Die Grunde, die C. Vogt, der ubrigens nach seinen eigenen Angaben (p. 210) núr Schadelansgusse, keine Gehirne untersuchen konnte, anführt, um die Annaherung an den Affentypus wenigstens für die eigentlichen Hemisphärentheile zu beweisen, sind wesentlich drei. Ich lasse sie der Reihe nach folgen und werde an jeden derselben sofort die Kritik anknupfen:

1) Der Siebbeinschnabel ist viel starker entwickelt als beim normalen Gehirn - ganz wie bei den anthropomorphen Affen. - Dies ist bedingt richtig. Ich habe jedoch schon darauf aufmerksam gemacht, dass Uebergange dazu auch an sonst normalen Gehirnen sich leicht auffinden lassen.

2) Die Sylvische Spalte hat bei normalen Menschengehirnen die Gestalt eines $\mathrm{Y}, \mathrm{d}$. h. sie besitzt einen gemeinschaftlichen Stamm, der sich erst nach oben hin gabelformig theilt. Die vordere Begranzung wird von dem orbitalen Theil der 3. Stirnwindung, die hintere von der Spitze des Schlafenlappens gebildet. Zwischen beide schiebt sich der Klappdeckel, die Gabelung bedingend, so ein, dass er die Basis nicht erreicht. Bei den Affen erreicht er die Basis und dadurch bekommt die Fossa Sylvii die Gestalt eines $\mathrm{V}$, d. h. sie hat gar keinen gemeinschaftlichen Stamm, sondern geht sofort in zwei Schenkel auseinander. So soll es sich auch bei allen Microcephalen verhalten (p. 233). - Dies ist nicht richtig; man braucht nur meine Figuren I. und IV. zu vergleichen, um sofort zu sehen, dass die Fossa Sylvii ebenso einen gemeinsamen Stamm hat, wie beim normalen Gehirn. Andentungen eines gemeinsamen Stammes zeigen sich auch in den beiden von Gratiolet abgebildeten Gehirnen (Anatomie comp. du syst. nerv. Atl. pl. 24 und 32); fur Vogt spricht nur die Abbildung bei Wagner (1. c. Tab. III., Fig. 3, vergl. auch Thoile, uber Mierocephalie; Archiv von Henle und Pfeuffer, XI., p. 211, Tab. X. und XI.). So viel steht also fest, dass Vogt's Angabe keineswegs für alle Falle stichhaltig ist. Dagegen existirt auch in meinen beiden Fallen die Verbindungsbrucke zwischen vorderer Central- und 3. Stirnwindung. Natürlich muss es für die ganze Bildungsgeschichte der Hemispharen von 
grosser Wichtigkeit sein, wie der Klappdeckel im gegebenen Fall beschaffen ist. Bekauntlich uberwachst derselbe erst in einer spateren foetalen Periode den ursprünglich frei liegenden Stammlappen. Es ist gewiss nicht im Sinne einer Hemmungsbildung, wenn der Klappdeckel nicht nur normal weit nach unten herabreicht, sondern sogar weiter (wic in dem Theile-Wagner'schen Fall), und spricht fur eine Entwickelung in einer ganz anderen Richtung, die noch der Aufklarung dnreh fernere Untersuchungen bedarf. Es sind bis jetzt so wenig Gehirne von Microcephalen beschrieben und brauchbar abgebildet worden, dass unan vorlaufig abwarten muss, in wie weit hier eine abweichende Bildung haufiger vorkommt. -

3) Ganz besonders betont Vogt, dass er im Gegensatz zu den Angaben Wagner's durch Messung der Oberflachen gefunden habe, dass die lobi oceipitales keineswegs besonders und stäker als dic ubrigen Lappen verkümmert seien; sie seien nur conform der Verkümmerung der andern Lappen gleichfalls verkleinert, ja sie seien verhaltnissmassig besser entwickelt als die Scheitellappen. - Hicrmit kann ich mich in keiner Weise einverstauden erklaren; Wagner hat vollkommen Recht. Die Hinterlappen sind ungemein stark verkümmert, so stark, dass sic in meinen beiden Fallen eigentlich ganz bis auf den kleinen Zwickel verschwunden sind. Ich habe von Messungen Abstand genommen, da ich nicht im Stande war, sic auch nur annahernd gegen die Scheitellappen abzugrenzen. Wie wenig Vertrauen ibligens an Schadelausgussen vorgenommene Messungen verdienen, beweist $V$ og t's Tafel X., wosclbst er, mit D. bezeichnet, einen Theil des Cerebellum als lobus orcipitalis deutet. Ich kann nur an Gehirnen ausgefuhrte Untersnchnngen anerkenuen. Uebrigens kann ich fiur meine Meinung einen Umstund anfuhren, der bis jetyt ubersehen zu sein scheint; nicht nur in meinen beiden, sondern auch in dem von Gratiolet (Tab. 32) abgchildeten Falle ist das Splenium des Balkens sehr stark verdunnt und diese Verdunnung des Splenium hängt zusammen mit mangelhafter Ausbildung der Hinterlappen, wic ich dies ausführlich in einer in 1. Heft dieses Archivs enthaltenen Arbeit ,uber Balkenmangel im menschlichen Gehirn" auscinandergesetzt habe. - Es mochte ausserdem nicht ohne Bedenken sein, den lobus occipitalis ohne Weiteres als gleichwerthig mit den anderen Grosshirnlappen anzusehen, wenn man sich erinnert, dass derselbe in der ursprunglichen Anlage des Grosshirns fehlt und erst spater ans derselben hervorsprosst.

Andere Punkte übergehe ich, wie z. B. die Anordnung der Win. dungen im Pli courbe (Gratiolet). Dieselbe ist schon bei sonst 
normalen Gehirnen eine so verschiedene, dass es mir bis jetzt nicht möglich gewesen ist, ein bestimmtes Gesetz darin zu finden.

Ich glaube hinreichend gezeigt zu haben, dass die Aehnlichkeit des Microcephalen-Gehirns mit dem Affengehirn eine unbewiesene Annahme ist, nur auf den äusseren Schein begründet; ich sehe in ersterem ein fehlerhaft entwickeltes Menschengehirn, dessen Bildungsgesetz noch aufzusuchen (vergl. fur den Schädel $\mathrm{Aeby}$, die Schadelformen etc., I eipzig 1867, p. 88, der zu ähnlichen Schlüssen kommt). Damit hängt auch die Frage zusammen: in welcher Zeit des fötalen Lebens beginnt die Storung? Zur Beantwortung derselben fehlt es noch ganz an Material. Dass die Commissuren normal ausgebildet sind, beweist noch nicht, dass erst nach erfolgtem Commissurenschluss die fehlerhafte Bildung begann; es ist ganz gut möglich, dass in ihrem Wachsthum sonst schon tief gestörte Hemispharen doch in der Mittellinie sich normal entgegenkommen können. Es ist natürlich werthlos, Vermuthungen aufzustellen; allein die Auffindung der Storung in ihrem ersten Beginn kann die Frage lösen.

\section{Tafel - Erklärung.}

Sämmtliche Figuren verdanke tch meinem werthen Freunde, Herrn Dr. W. Dönitz, Assistenten am hiesigen anatomischen Institut. Fig. III. und IV. sind mittelst des Lucae'schen Apparates gezeichnet.

Fig. I. Linke Hemisphàre des Pfefferle von der lateralen Seite

A. Stimlappen. g'esehen.

B. Scheitellappen.

C. Schläfelappen.

D. Hintere Spitze der Hemisphare.

d. Hirnstock.

I. Fissura Rolandi.

II. Sulcus occipitalis (?).

III. Fissura parallela.

1. 2. 3. 1., 2. und 3. Stirnwindung.

a. Vordere Centralwindung.

b. Hintere Centralwindung.

a. Spitze des Klappdeckels und Verbindungsbrücke zwischen a und 3 .

c. Oberer Theil der hinteren Centralwindung, der in Veraindung steht mit

e und $\mathrm{f}$ dem Vorzwickel.

7. pli courbe (zweite Parietalwindung).

4. Erster gyrus sphenoidalis.

5. Zweiter "

6. Dritter " $"$

g. Zwickel.

y. Fossa Sylvii. 
Fig. II. Dieselbe Hemisphäre von ihrer medialen Seite. A, B, D, d wie in Fig. I. th. o. thalamus opticus.

t. chor. sup. tela choreoidea superior mit dem Plexus choreoideus.

l. t. lamina terminalis

c. a. commissura anterior.

s. l. septum pellucidum.

c. call. corpus callosum.

gen. genu.

spl. splenium.

forn. Fornix.

g. forn. gyrus fornicatus.

h. und i. Windungen des lobus occipitalis (?).

Fig. III. Hirn des Friedrich Sohn von oben gesehen.

$A, B, D$, wie fr ùher.

I. Fissura Rolandi.

II. Fissura parallela.

a. Vordere Centralwindung.

1. 2. 3. Stirnwindungen.

b. Hintere Centralwindung.

e. Vorzwickel.

f. Zweite Parietalwindung (pli courbe).

g. Zwickel.

Fig. IV. Dasselbe von der linken Seite.

$\mathrm{A}, \mathrm{B}, \mathrm{C}, \mathrm{D}$, wie fruher.

E. Cerebellum.

I. und II, wie früher.

1. 2. 3. Stirnwindungen.

a. und b. Centralwindungen.

ж. Spitze des Klappdeckels.

$\beta$. Verbindungsbrucke zwsschen vordeses Central- und 3. Stirnwindung.

y. Fossa Sylvi1,

4. Erster gyrus sphenoidalis.

5. Zweiter

6. Dritter "

6. Dritter " $"$

e, $f, g$, wie frùher.

Berlin, im December 1867. 